\title{
The real evidence for polymyalgia rheumatic as a paraneoplastic syndrome
}

\author{
S. Muller ${ }^{1}$, S. Hider ${ }^{1,2}$, T. Helliwell ${ }^{1}$, R. Partington ${ }^{1}$, C. Mallen ${ }^{1}$ \\ 'Institute for Primary Care \& Health Sciences, Keele University, UK; \\ ${ }^{2}$ Haywood Academic Rheumatology Centre, Staffordshire and Stoke on Trent Partnership Trust, UK
}

$\overline{\text { Corresponding author: }}$ Sara Muller Institute for Primary Care $\&$ Health Sciences, David Weatherall Building. Keele University, UK ST5 5BG E-mail: s.muller@keele.ac.uk
$\mathrm{D}$ ear Editor,

We would like to thank Bellan and colleagues for their interest in our recent article regarding polymyalgia rheumatica (PMR) and paraneoplastic syndrome (1, $2)$. We agree with the points they raise and are grateful for the aspects they highlight regarding the need for further research into PMR.

First, we agree that the definition of paraneoplastic syndrome is controversial, as indeed is the definition of PMR. With no clear diagnostic test for PMR, and classification criteria (3) developed for use in trials rather than clinical practice, there is undoubtedly some heterogeneity in what is diagnosed as PMR. This is likely amplified by the comparison of samples recruited in different settings [e.g. tertiary care (4), primary care (5)]. Furthermore, Bellan and colleagues (2) highlight the related point of our exclusion of existing cancer in our previous study (5). Whilst we may accept that if PMR were a paraneoplastic syndrome, it does not need to occur before the malignancy is diagnosed, British guidelines for the diagnosis of PMR state that malignancy is an important differential diagnosis of polymyalgia symptoms and hence should be excluded as the cause of symptoms before a diagnosis of PMR is made. Given that clinicians may then undertake additional investigations (e.g. chest Xray, prostate specific antigen) to look for malignancy (and exclude this) in patients with PMR, this leads to additional risks of surveillance bias and hence our decision to exclude pre-existing cancer in our earlier study. Again, this makes answer- ing the question as to whether PMR is a paraneoplastic syndrome or a differential diagnosis of malignancy problematic.

Second, the issue of the length of follow-up required is interesting. It is likely that studies based in routinely collected data have less detail than those for which data were collected specifically for research. However, they are likely to contain major diagnoses, such as cancer, and in many cases, they are likely to have a longer follow-up, which may have the effect of increasing the likelihood of a later diagnosis of malignancy being recorded during period under study. However, this may also mean that any short-term association with cancer is diluted by a lower risk of malignancy in the longer term. Indeed, the two studies in our review that specifically looked at intervals of time from PMR diagnosis showed an initial increase in the risk of malignancy, followed by a return to normal risk $(5,6)$. Finally, we agree that a specific cohort study may well be the only way to definitively answer the question as to whether PMR is a truly paraneoplastic condition. However, this would still require clearly agreed definitions of both PMR and paraneoplastic syndrome. Furthermore, our experience of recruiting a representative, prospective PMR cohort in England suggests that whilst this is possible, it is not easy $(7,8)$.

Undoubtedly whether or not PMR is considered truly paraneoplastic requires further study, however the temporal relationship with malignancy, whether as a differential diagnosis of PMR itself or as a paraneoplastic phenomenon, supports the need for physicians to actively con- 
sider (and screen for) malignancy, as per UK guidelines. We agree that only with prospectively conducted, long term cohort studies will we be able to accurately quantify the risks of malignancy and determine whether certain subgroups of patients are at particularly high risk of cancer or whether this approach is required for all.

\section{REFERENCES}

1. Muller S, Hider S, Helliwell T, et al. The real evidence for polymyalgia rheumatica as a paraneoplastic syndrome. Reumatismo. 2018; 70: 23-34.

2. Bellan M, Sainaghi PP, Pirisi M. Comment on the paper "The real evidence for polymyalgia rheumatic as a paraneoplastic syndrome". Reumatismo. 2018; 70: [In press].

3. Dasgupta B, Cimmino MA, Kremers HM, et al. 2012 Provisional classification criteria for polymyalgia rheumatica: a European League Against Rheumatism/American College of
Rheumatology collaborative initiative. Arthritis Rheum. 2012; 64: 943-954.

4. Bellan M, Boggio E, Sola D, et al. Association between rheumatic diseases and cancer: results from a clinical practice cohort study. Intern Emerg Med. 2017;12: 621-627.

5. Muller S, Hider SL, Belcher J, et al. Is cancer associated with polymyalgia rheumatica? A cohort study in the General Practice Research Database. Ann Rheum Dis. 2014; 73: 17691773.

6. Ji J, Liu X, Sundquist K, et al. Cancer risk in patients hospitalized with polymyalgia rheumatica and giant cell arteritis: a follow-up study in Sweden. Rheumatology (Oxford). 2010; 49: 1158-63.

7. Muller S, Hider S, Helliwell T, et al. The epidemiology of polymyalgia rheumatica in primary care: a research protocol. BMC Musculoskelet Disord. 2012; 13: 102.

8. Muller S, Hider SL, Helliwell T, et al. Characterising those with incident polymyalgia rheumatica in primary care: results from the PMR Cohort Study. Arthritis Res Ther. 2016; 18: 200. 